\title{
An Improved United-Atom Force Field for 1-Alkyl-3-methylimidazolium chloride
}

\author{
Zhiping Liu ${ }^{\ddagger}$, Ting Chen, Alex Bell,* Berend Smit* \\ (Energy Biosciences Institute and Department of Chemical Engineering, University of \\ California, Berkeley, Berkeley, CA 94720-1462, USA) \\ Email: alexbell@berkeley.edu, berend-smit@berkeley.edu
}

November 16, 2009

\begin{abstract}
Supporting information: All the parameters of the force fields for $\left[\mathrm{C}_{\mathrm{n}} \operatorname{mim}\right][\mathrm{Cl}]$ can be found in Table S1. The atom coordinates in the optimized geometries of monomers and dimers of $\left[\mathrm{C}_{1} \operatorname{mim}\right][\mathrm{Cl}]$ and $\left[\mathrm{C}_{2} \operatorname{mim}\right][\mathrm{Cl}]$ were given in Table $\mathrm{S} 2$ for reference. Figures $\mathrm{S} 1$ and $\mathrm{S} 2$ gave the similar results for $\left[\mathrm{C}_{2} \mathrm{mim}\right][\mathrm{Cl}]$, as those discussed in section 3 for $\left[\mathrm{C}_{1} \mathrm{mim}\right][\mathrm{Cl}]$. The typical distributions of counter-ions around the center anion and cation were demonstrated by the snapshots in Figs. S3 and S4. Figure S5 gave a typical integral of correlation function to estimate the viscosity。
\end{abstract}


Table S1 Force field parameters in this work

Non-bonded

\begin{tabular}{c|cc|cc}
\hline & \multicolumn{2}{|c|}{ Lennard-Jones } & \multicolumn{2}{c}{ charges / lel * } \\
\hline Atom & $\sigma / \AA$ & $\varepsilon / \mathrm{kcal}_{\mathrm{mol}}{ }^{-1}$ & {$\left[\mathrm{C}_{1} \mathrm{mim}\right][\mathrm{Cl}]$} & {$\left[\mathrm{C}_{\mathrm{n}} \mathrm{mim}\right][\mathrm{Cl}](\mathrm{n} \geq 2)$} \\
\hline $\mathrm{NA}$ & 3.250 & 0.170 & 0.027 & 0.015 \\
$\mathrm{CR}$ & 3.400 & 0.086 & 0.005 & 0.00 \\
$\mathrm{CW}$ & 3.400 & 0.086 & -0.1734 & -0.16 \\
$\mathrm{H} 5$ & 1.247 & 0.030 & 0.1544 & 0.15 \\
$\mathrm{H} 4$ & 1.604 & 0.030 & 0.2188 & 0.20 \\
$\mathrm{CN} 3$ & 3.813 & 0.190 & 0.2543 & 0.26 \\
$\mathrm{CN} 2$ & 3.822 & 0.142 & 0.2543 & 0.23 \\
$\mathrm{CT} 2$ & 3.947 & 0.133 & - & $0.03^{*}$ \\
$\mathrm{CT} 3$ & 3.902 & 0.185 & - & 0.00 \\
$\mathrm{Cl}$ & 3.742 & 0.150 & -0.80 & -0.80 \\
\hline
\end{tabular}

* The value is for CT2 connected with CN2. For other CT2, the charges are set to zero.

\begin{tabular}{l|ll}
\multicolumn{3}{l}{ Bonds } \\
\hline Bond & $\mathrm{k}_{\mathrm{r}} /{\mathrm{kcal} . \mathrm{mol}^{-1} \AA^{-2}}^{-2} \mathrm{r}_{0} / \AA$ \\
\hline NA-CR & 411.1 & 1.325 \\
CW-NA & 411.1 & 1.378 \\
CW-CW & 478.4 & 1.343 \\
CW-H4 & 344.3 & 1.070 \\
CR-H5 & 344.3 & 1.070 \\
NA-CN2 & 337.0 & 1.472 \\
CT2-CN2 & 310.0 & 1.526 \\
\hline
\end{tabular}

\begin{tabular}{l|ll}
\multicolumn{3}{l}{ Angles } \\
\hline Angle & $\mathrm{k}_{\theta} / \mathrm{kcal}^{-\mathrm{mol}^{-1} \mathrm{rad}^{-2}}$ & $\theta_{0} / \mathrm{rad}$ \\
\hline CW-NA-CR & 67.8 & 108.0 \\
CW-NA-CN2 & 64.2 & 125.7 \\
CR-NA-CN2 & 64.2 & 126.3 \\
NA-CR-NA & 73.7 & 109.9 \\
NA-CR-H5 & 51.2 & 125.7 \\
CN3-NA-CW & 64.2 & 125.7 \\
CN3-NA-CR & 64.2 & 126.3 \\
CW-NA-CR & 67.8 & 108.0 \\
H4-CW-NA & 51.2 & 122.1 \\
H4-CW-CW & 50.0 & 130.7 \\
NA-CW-CW & 69.8 & 107.1 \\
CW-CW-H4 & 50.0 & 130.7 \\
CW-CW-NA & 69.8 & 107.1 \\
H4-CW-NA & 51.2 & 122.1 \\
CT2-CN2-NA & 80.0 & 112.2 \\
CT2-CT2-CN2 & 40.0 & 109.5 \\
\hline
\end{tabular}


Dihedrals

\begin{tabular}{l|lll}
\hline Dihedral & $\mathrm{n} \mathrm{k}_{\chi} / \mathrm{kcal}^{-m_{1}}{ }^{-1}$ & $\delta /$ degree \\
\hline H4-CW-NA-CN3 & 2 & 1.5 & 180 \\
H4-CW-NA-CR & 2 & 2 & 180 \\
CW-CW-NA-CR & 2 & 12 & 180 \\
NA-CW-CW-H4 & 2 & 1.5 & 180 \\
NA-CW-CW-NA & 2 & 12 & 180 \\
CN3-NA-CR-NA & 2 & 2 & 180 \\
CN3-NA-CR-H5 & 2 & 1.5 & 180 \\
CW-NA-CR-NA & 2 & 12 & 180 \\
CW-NA-CR-H5 & 2 & 1.5 & 180 \\
CW-CW-NA-CR & 2 & 12 & 180 \\
CW-CW-NA-CN2 & 2 & 2 & 180 \\
H4-CW-NA-CR & 2 & 2 & 180 \\
H4-CW-NA-CN2 & 2 & 1.5 & 180 \\
NA-CR-NA-CW & 2 & 12 & 180 \\
NA-CR-NA-CN2 & 2 & 2 & 180 \\
H5-CR-NA-CW & 2 & 1.5 & 180 \\
H5-CR-NA-CN2 & 2 & 1.5 & 180 \\
CW-NA-CN2-CT2 & 1 & 0.694 & 0 \\
& 2 & 0.554 & 180 \\
CR-NA-CN2-CT2 & 3 & -0.564 & 0 \\
& 2 & 0.518 & 0 \\
X-CT2-CT2-X & 1 & 0.046 & 180 \\
& 2 & -0.782 & 0 \\
& 3 & 1.6 & 0 \\
& & 180 \\
& & 0 \\
\hline
\end{tabular}


Table S2a Atom coordinates (unit: $0.1 \mathrm{~nm}$ ) in optimized geometry of $\left[\mathrm{C}_{1} \mathrm{mim}\right][\mathrm{Cl}]$ monomers at B3LYP/6-31G+(d,p) level

\begin{tabular}{|c|c|c|c|c|c|c|c|c|c|}
\hline & Atoms & $\mathrm{X}$ & Y & Z & & Atoms & $\mathrm{X}$ & $\mathrm{Y}$ & Z \\
\hline \multirow{17}{*}{$5 m$} & $\mathrm{~N}$ & 1.309 & -0.832 & 0.000 & \multirow{17}{*}{$4 m$} & $\mathrm{~N}$ & -2.070 & -0.244 & 0.000 \\
\hline & $\mathrm{C}$ & 0.145 & -0.165 & 0.000 & & $\mathrm{C}$ & -1.461 & 0.953 & 0.000 \\
\hline & $\mathrm{N}$ & 0.423 & 1.146 & 0.000 & & $\mathrm{~N}$ & -0.137 & 0.759 & 0.000 \\
\hline & $\mathrm{C}$ & 1.796 & 1.322 & 0.000 & & $\mathrm{C}$ & 0.124 & -0.600 & 0.000 \\
\hline & $\mathrm{C}$ & 2.357 & 0.078 & 0.000 & & $\mathrm{C}$ & -1.091 & -1.226 & 0.000 \\
\hline & $\mathrm{H}$ & -0.908 & -0.560 & 0.000 & & $\mathrm{H}$ & -1.960 & 1.909 & 0.000 \\
\hline & $\mathrm{H}$ & 2.252 & 2.299 & 0.000 & & $\mathrm{H}$ & 1.176 & -0.939 & 0.000 \\
\hline & $\mathrm{H}$ & 3.390 & -0.230 & 0.000 & & $\mathrm{H}$ & -1.340 & -2.275 & 0.000 \\
\hline & $\mathrm{C}$ & -0.610 & 2.193 & 0.000 & & $\mathrm{C}$ & 0.898 & 1.814 & 0.000 \\
\hline & $\mathrm{C}$ & 1.422 & -2.292 & 0.000 & & $\mathrm{C}$ & -3.515 & -0.470 & 0.000 \\
\hline & $\mathrm{H}$ & -1.585 & 1.692 & 0.000 & & $\mathrm{H}$ & 0.787 & 2.426 & -0.897 \\
\hline & $\mathrm{H}$ & -0.501 & 2.809 & 0.895 & & $\mathrm{H}$ & 0.787 & 2.426 & 0.898 \\
\hline & $\mathrm{H}$ & -0.501 & 2.809 & -0.895 & & $\mathrm{H}$ & 1.873 & 1.302 & 0.000 \\
\hline & $\mathrm{H}$ & 0.416 & -2.712 & 0.000 & & $\mathrm{H}$ & -4.028 & 0.492 & 0.000 \\
\hline & $\mathrm{H}$ & 1.955 & -2.623 & 0.894 & & $\mathrm{H}$ & -3.801 & -1.030 & 0.893 \\
\hline & $\mathrm{H}$ & 1.955 & -2.623 & -0.894 & & $\mathrm{H}$ & -3.801 & -1.030 & -0.893 \\
\hline & $\mathrm{Cl}$ & -2.897 & -0.581 & 0.000 & & $\mathrm{Cl}$ & 3.296 & -0.571 & 0.000 \\
\hline \multirow{17}{*}{44} & $\mathrm{~N}$ & 1.129 & 1.088 & 0.000 & \multirow{17}{*}{ top } & $\mathrm{N}$ & 1.092 & -0.743 & 0.269 \\
\hline & $\mathrm{C}$ & 1.917 & 0.000 & 0.000 & & $\mathrm{C}$ & 0.000 & -0.158 & 0.794 \\
\hline & $\mathrm{N}$ & 1.129 & -1.088 & 0.000 & & $\mathrm{~N}$ & -1.092 & -0.743 & 0.269 \\
\hline & $\mathrm{C}$ & -0.194 & -0.682 & 0.000 & & $\mathrm{C}$ & -0.680 & -1.617 & -0.728 \\
\hline & $\mathrm{C}$ & -0.194 & 0.682 & 0.000 & & $\mathrm{C}$ & 0.680 & -1.617 & -0.728 \\
\hline & $\mathrm{H}$ & 2.995 & 0.000 & 0.000 & & $\mathrm{H}$ & 0.000 & 0.526 & 1.623 \\
\hline & $\mathrm{H}$ & -1.066 & -1.326 & 0.000 & & $\mathrm{H}$ & -1.384 & -2.163 & -1.333 \\
\hline & $\mathrm{H}$ & -1.067 & 1.325 & 0.000 & & $\mathrm{H}$ & 1.384 & -2.163 & -1.333 \\
\hline & $\mathrm{C}$ & 1.587 & -2.477 & 0.000 & & $\mathrm{C}$ & -2.443 & -0.215 & 0.446 \\
\hline & $\mathrm{C}$ & 1.587 & 2.478 & 0.000 & & $\mathrm{C}$ & 2.443 & -0.215 & 0.446 \\
\hline & $\mathrm{H}$ & 2.678 & -2.498 & 0.000 & & $\mathrm{H}$ & -2.664 & -0.117 & 1.511 \\
\hline & $\mathrm{H}$ & 1.213 & -2.984 & 0.892 & & $\mathrm{H}$ & -2.491 & 0.767 & -0.035 \\
\hline & $\mathrm{H}$ & 1.213 & -2.984 & -0.892 & & $\mathrm{H}$ & -3.154 & -0.909 & -0.004 \\
\hline & $\mathrm{H}$ & 2.677 & 2.498 & 0.000 & & $\mathrm{H}$ & 2.664 & -0.117 & 1.511 \\
\hline & $\mathrm{H}$ & 1.212 & 2.984 & 0.892 & & $\mathrm{H}$ & 2.491 & 0.767 & -0.035 \\
\hline & $\mathrm{H}$ & 1.212 & 2.985 & -0.891 & & $\mathrm{H}$ & 3.154 & -0.908 & -0.004 \\
\hline & $\mathrm{Cl}$ & -3.240 & 0.000 & 0.000 & & $\mathrm{Cl}$ & 0.000 & 2.215 & -0.415 \\
\hline
\end{tabular}


Table S2a Atom coordinates (unit: $0.1 \mathrm{~nm}$ ) in optimized geometry of $\left[\mathrm{C}_{2} \mathrm{mim}\right][\mathrm{Cl}]$ monomers at B3LYP/6-31G+(d,p) level

\begin{tabular}{|c|c|c|c|c|c|c|c|c|c|}
\hline & Atoms & $\mathrm{X}$ & Y & Z & & Atoms & $\mathrm{X}$ & Y & Z \\
\hline \multirow{20}{*}{$5 e$} & $\mathrm{~N}$ & -0.251 & 0.912 & -0.286 & \multirow{20}{*}{$5 \mathrm{~m}$} & $\mathrm{~N}$ & 1.293 & 0.405 & -0.166 \\
\hline & $\mathrm{C}$ & -0.352 & -0.403 & -0.048 & & $\mathrm{C}$ & 0.097 & -0.201 & -0.118 \\
\hline & $\mathrm{N}$ & -1.655 & -0.702 & 0.075 & & $\mathrm{~N}$ & 0.305 & -1.514 & 0.041 \\
\hline & $\mathrm{C}$ & -2.405 & 0.454 & -0.088 & & $\mathrm{C}$ & 1.667 & -1.759 & 0.091 \\
\hline & $\mathrm{C}$ & -1.520 & 1.468 & -0.313 & & $\mathrm{C}$ & 2.289 & -0.552 & -0.042 \\
\hline & $\mathrm{H}$ & 0.545 & -1.078 & 0.006 & & $\mathrm{H}$ & -0.932 & 0.246 & -0.169 \\
\hline & $\mathrm{H}$ & -3.482 & 0.456 & -0.035 & & $\mathrm{H}$ & 2.072 & -2.750 & 0.221 \\
\hline & $\mathrm{H}$ & -1.686 & 2.518 & -0.492 & & $\mathrm{H}$ & 3.337 & -0.297 & -0.054 \\
\hline & $\mathrm{C}$ & -2.171 & -2.047 & 0.333 & & $\mathrm{C}$ & -0.785 & -2.495 & 0.151 \\
\hline & $\mathrm{C}$ & 1.041 & 1.612 & -0.466 & & $\mathrm{C}$ & 1.485 & 1.857 & -0.373 \\
\hline & $\mathrm{C}$ & 1.404 & 2.464 & 0.748 & & $\mathrm{C}$ & 0.564 & 2.696 & 0.509 \\
\hline & $\mathrm{H}$ & -2.825 & -2.357 & -0.485 & & $\mathrm{H}$ & -0.701 & -3.026 & 1.101 \\
\hline & $\mathrm{H}$ & -1.324 & -2.731 & 0.398 & & $\mathrm{H}$ & -1.729 & -1.942 & 0.110 \\
\hline & $\mathrm{H}$ & -2.723 & -2.060 & 1.275 & & $\mathrm{H}$ & -0.724 & -3.202 & -0.680 \\
\hline & $\mathrm{H}$ & 0.956 & 2.216 & -1.374 & & $\mathrm{H}$ & 1.312 & 2.069 & -1.433 \\
\hline & $\mathrm{H}$ & 1.785 & 0.823 & -0.624 & & $\mathrm{H}$ & 2.537 & 2.060 & -0.157 \\
\hline & $\mathrm{H}$ & 1.519 & 1.836 & 1.636 & & $\mathrm{H}$ & 0.758 & 2.508 & 1.569 \\
\hline & $\mathrm{H}$ & 2.362 & 2.959 & 0.562 & & $\mathrm{H}$ & 0.750 & 3.755 & 0.307 \\
\hline & $\mathrm{H}$ & 0.655 & 3.237 & 0.952 & & $\mathrm{H}$ & -0.492 & 2.491 & 0.306 \\
\hline & $\mathrm{Cl}$ & 2.446 & -1.681 & -0.078 & & $\mathrm{Cl}$ & -2.899 & 0.505 & -0.091 \\
\hline \multirow{20}{*}{$4 e$} & $\mathrm{~N}$ & -0.225 & 0.485 & -0.257 & \multirow{20}{*}{$4 m$} & $\mathrm{~N}$ & 1.637 & 0.022 & -0.336 \\
\hline & $\mathrm{C}$ & -1.525 & 0.777 & -0.359 & & $\mathrm{C}$ & 0.946 & 1.149 & -0.102 \\
\hline & $\mathrm{N}$ & -2.239 & -0.300 & 0.006 & & $\mathrm{~N}$ & -0.356 & 0.852 & -0.043 \\
\hline & $\mathrm{C}$ & -1.350 & -1.308 & 0.354 & & $\mathrm{C}$ & -0.521 & -0.508 & -0.244 \\
\hline & $\mathrm{C}$ & -0.085 & -0.818 & 0.191 & & $\mathrm{C}$ & 0.731 & -1.025 & -0.425 \\
\hline & $\mathrm{H}$ & -1.938 & 1.718 & -0.685 & & $\mathrm{H}$ & 1.376 & 2.132 & 0.015 \\
\hline & $\mathrm{H}$ & -1.691 & -2.279 & 0.674 & & $\mathrm{H}$ & -1.542 & -0.930 & -0.214 \\
\hline & $\mathrm{H}$ & 0.932 & -1.237 & 0.292 & & $\mathrm{H}$ & 1.050 & -2.038 & -0.611 \\
\hline & $\mathrm{C}$ & -3.698 & -0.398 & 0.019 & & $\mathrm{C}$ & -1.460 & 1.807 & 0.183 \\
\hline & $\mathrm{C}$ & 0.902 & 1.410 & -0.543 & & $\mathrm{C}$ & 3.102 & -0.086 & -0.426 \\
\hline & $\mathrm{C}$ & 1.335 & 2.176 & 0.703 & & $\mathrm{C}$ & 3.725 & -0.693 & 0.831 \\
\hline & $\mathrm{H}$ & -4.043 & -0.633 & 1.028 & & $\mathrm{H}$ & -1.277 & 2.353 & 1.110 \\
\hline & $\mathrm{H}$ & -4.124 & 0.556 & -0.292 & & $\mathrm{H}$ & -1.519 & 2.496 & -0.662 \\
\hline & $\mathrm{H}$ & -4.023 & -1.179 & -0.671 & & $\mathrm{H}$ & -2.385 & 1.213 & 0.256 \\
\hline & $\mathrm{H}$ & 0.566 & 2.075 & -1.344 & & $\mathrm{H}$ & 3.487 & 0.921 & -0.610 \\
\hline & $\mathrm{H}$ & 1.727 & 0.772 & -0.887 & & $\mathrm{H}$ & 3.328 & -0.689 & -1.310 \\
\hline & $\mathrm{H}$ & 1.724 & 1.481 & 1.452 & & $\mathrm{H}$ & 3.350 & -1.704 & 1.013 \\
\hline & $\mathrm{H}$ & 2.150 & 2.854 & 0.432 & & $\mathrm{H}$ & 4.810 & -0.750 & 0.707 \\
\hline & $\mathrm{H}$ & 0.519 & 2.769 & 1.132 & & $\mathrm{H}$ & 3.510 & -0.082 & 1.712 \\
\hline & $\mathrm{Cl}$ & 3.057 & -1.131 & -0.093 & & $\mathrm{Cl}$ & -3.664 & -0.760 & 0.138 \\
\hline
\end{tabular}


Table S2c Atom coordinates (unit: $0.1 \mathrm{~nm}$ ) in optimized geometry of $\left[\mathrm{C}_{1} \mathrm{mim}\right][\mathrm{Cl}]$ dimer at B3LYP/6-31G+(d,p) level

\begin{tabular}{|c|c|c|c|c|c|}
\hline & Atoms & $X$ & $\mathrm{Y}$ & $\mathrm{Z}$ & Atom type \\
\hline 1 & N1 & 3.067 & -0.357 & 0.198 & NA \\
\hline 2 & $\mathrm{C} 1$ & 2.206 & -0.212 & -0.819 & $\mathrm{CR}$ \\
\hline 3 & $\mathrm{~N} 2$ & 1.534 & -1.361 & -0.967 & NA \\
\hline 4 & $\mathrm{C} 2$ & 1.965 & -2.265 & -0.012 & $\mathrm{CA}$ \\
\hline 5 & $\mathrm{C} 3$ & 2.938 & -1.638 & 0.711 & $\mathrm{CA}$ \\
\hline 6 & $\mathrm{H} 1$ & 2.009 & 0.739 & -1.316 & H5 \\
\hline 7 & $\mathrm{H} 2$ & 1.489 & -3.225 & 0.109 & $\mathrm{H} 4$ \\
\hline 8 & $\mathrm{H} 3$ & 3.522 & -1.980 & 1.551 & $\mathrm{H} 4$ \\
\hline 9 & $\mathrm{C} 4$ & 0.484 & -1.612 & -1.959 & $\mathrm{CT}$ \\
\hline 10 & C5 & 3.961 & 0.704 & 0.677 & $\mathrm{CT}$ \\
\hline 11 & $\mathrm{H} 4$ & 0.194 & -0.658 & -2.401 & $\mathrm{H} 1$ \\
\hline 12 & H5 & 0.868 & -2.276 & -2.738 & $\mathrm{H} 1$ \\
\hline 13 & H6 & -0.361 & -2.076 & -1.437 & $\mathrm{H} 1$ \\
\hline 14 & $\mathrm{H} 7$ & 3.535 & 1.664 & 0.370 & $\mathrm{H} 1$ \\
\hline 15 & H8 & 4.960 & 0.572 & 0.253 & $\mathrm{H} 1$ \\
\hline 16 & H9 & 4.014 & 0.654 & 1.766 & $\mathrm{H} 1$ \\
\hline 17 & N3 & -3.067 & 0.357 & -0.198 & NA \\
\hline 18 & C6 & -2.206 & 0.212 & 0.819 & $\mathrm{CR}$ \\
\hline 19 & N4 & -1.534 & 1.361 & 0.967 & NA \\
\hline 20 & $\mathrm{C} 7$ & -1.965 & 2.265 & 0.012 & $\mathrm{CA}$ \\
\hline 21 & $\mathrm{C} 8$ & -2.938 & 1.638 & -0.711 & $\mathrm{CA}$ \\
\hline 22 & H10 & -2.009 & -0.739 & 1.316 & H5 \\
\hline 23 & H11 & -1.489 & 3.225 & -0.109 & $\mathrm{H} 4$ \\
\hline 24 & $\mathrm{H} 12$ & -3.522 & 1.980 & -1.551 & $\mathrm{H} 4$ \\
\hline 25 & C9 & -0.484 & 1.612 & 1.959 & $\mathrm{CT}$ \\
\hline 26 & $\mathrm{C} 10$ & -3.961 & -0.704 & -0.677 & $\mathrm{CT}$ \\
\hline 27 & H13 & -0.868 & 2.276 & 2.738 & H1 \\
\hline 28 & H14 & -0.194 & 0.658 & 2.401 & H1 \\
\hline 29 & H15 & 0.361 & 2.076 & 1.437 & $\mathrm{H} 1$ \\
\hline 30 & H16 & -3.535 & -1.664 & -0.370 & H1 \\
\hline 31 & H17 & -4.014 & -0.654 & -1.766 & $\mathrm{H} 1$ \\
\hline 32 & H18 & -4.960 & -0.572 & -0.253 & H1 \\
\hline 33 & $\mathrm{Cl} 1$ & -1.406 & -2.883 & 0.675 & $\mathrm{Cl}$ \\
\hline 34 & $\mathrm{Cl} 2$ & 1.406 & 2.883 & -0.675 & $\mathrm{Cl}$ \\
\hline
\end{tabular}


Table S2d Atom coordinates (unit: $0.1 \mathrm{~nm}$ ) in optimized geometry of $\left[\mathrm{C}_{2} \mathrm{mim}\right][\mathrm{Cl}]$ dimer at B3LYP/6-31G+(d,p) level

\begin{tabular}{|c|c|c|c|c|c|}
\hline & Atoms & $X$ & $\mathrm{Y}$ & $\mathrm{Z}$ & Atom type \\
\hline 1 & N1 & -3.411 & 0.841 & 0.130 & NA \\
\hline 2 & $\mathrm{C} 1$ & -2.531 & 0.264 & -0.702 & $\mathrm{CR}$ \\
\hline 3 & $\mathrm{~N} 2$ & -1.574 & 1.156 & -0.985 & NA \\
\hline 4 & $\mathrm{C} 2$ & -1.833 & 2.336 & -0.310 & $\mathrm{CA}$ \\
\hline 5 & $\mathrm{C} 3$ & -2.989 & 2.140 & 0.387 & $\mathrm{CA}$ \\
\hline 6 & $\mathrm{H} 1$ & -2.489 & -0.793 & -0.974 & H5 \\
\hline 7 & $\mathrm{H} 2$ & -1.135 & 3.160 & -0.334 & $\mathrm{H} 4$ \\
\hline 8 & $\mathrm{H} 3$ & -3.532 & 2.797 & 1.047 & $\mathrm{H} 4$ \\
\hline 9 & $\mathrm{C} 4$ & -0.432 & 0.902 & -1.871 & $\mathrm{CT}$ \\
\hline 10 & $\mathrm{C} 5$ & -4.553 & 0.169 & 0.783 & $\mathrm{CT}$ \\
\hline 11 & C6 & -5.062 & -1.038 & 0.002 & $\mathrm{CT}$ \\
\hline 12 & $\mathrm{H} 4$ & -0.284 & -0.179 & -1.926 & H1 \\
\hline 13 & H5 & -0.644 & 1.302 & -2.866 & H1 \\
\hline 14 & H6 & 0.440 & 1.401 & -1.436 & $\mathrm{H} 1$ \\
\hline 15 & $\mathrm{H} 7$ & -5.334 & 0.926 & 0.894 & $\mathrm{H} 1$ \\
\hline 16 & H8 & -4.229 & -0.133 & 1.785 & $\mathrm{H} 1$ \\
\hline 17 & H9 & -5.923 & -1.456 & 0.532 & $\mathrm{HC}$ \\
\hline 18 & H10 & -5.388 & -0.752 & -1.003 & $\mathrm{HC}$ \\
\hline 19 & H11 & -4.301 & -1.821 & -0.075 & $\mathrm{HC}$ \\
\hline 20 & N3 & 3.410 & -0.841 & -0.131 & NA \\
\hline 21 & $\mathrm{C} 7$ & 2.531 & -0.264 & 0.702 & $\mathrm{CR}$ \\
\hline 22 & N4 & 1.574 & -1.156 & 0.985 & NA \\
\hline 23 & $\mathrm{C} 8$ & 1.833 & -2.336 & 0.310 & $\mathrm{CA}$ \\
\hline 24 & $\mathrm{C} 9$ & 2.989 & -2.140 & -0.387 & $\mathrm{CA}$ \\
\hline 25 & $\mathrm{C} 10$ & 5.062 & 1.038 & -0.003 & $\mathrm{CT}$ \\
\hline 26 & H12 & 2.488 & 0.793 & 0.973 & H5 \\
\hline 27 & H13 & 1.135 & -3.161 & 0.334 & $\mathrm{H} 4$ \\
\hline 28 & H14 & 3.532 & -2.797 & -1.047 & $\mathrm{H} 4$ \\
\hline 29 & C11 & 0.432 & -0.902 & 1.871 & $\mathrm{CT}$ \\
\hline 30 & C12 & 4.552 & -0.169 & -0.783 & $\mathrm{CT}$ \\
\hline 31 & H15 & 0.644 & -1.302 & 2.866 & H1 \\
\hline 32 & H16 & 0.284 & 0.178 & 1.927 & $\mathrm{H} 1$ \\
\hline 33 & H17 & -0.440 & -1.401 & 1.436 & $\mathrm{H} 1$ \\
\hline 34 & H18 & 4.229 & 0.133 & -1.785 & H1 \\
\hline 35 & H19 & 5.334 & -0.926 & -0.894 & H1 \\
\hline 36 & $\mathrm{H} 20$ & 5.388 & 0.752 & 1.003 & $\mathrm{HC}$ \\
\hline 37 & $\mathrm{H} 21$ & 5.923 & 1.456 & -0.532 & $\mathrm{HC}$ \\
\hline 38 & $\mathrm{H} 22$ & 4.300 & 1.821 & 0.075 & $\mathrm{HC}$ \\
\hline 39 & $\mathrm{Cl} 1$ & 1.535 & 2.754 & 0.408 & $\mathrm{Cl}$ \\
\hline 40 & $\mathrm{Cl} 2$ & -1.535 & -2.754 & -0.408 & $\mathrm{Cl}$ \\
\hline
\end{tabular}


Table S3 Atom charges of $\left[\mathrm{C}_{\mathrm{n}} \mathrm{mim}\right][\mathrm{Cl}](\mathrm{n}=1,3)$ by RESP fitting of ion-pair dimers

\begin{tabular}{c|ccc}
\hline & {$\left[\mathrm{C}_{1} \mathrm{mim}\right][\mathrm{Cl}]$} & {$\left[\mathrm{C}_{2} \mathrm{mim}\right][\mathrm{Cl}]$} & {$\left[\mathrm{C}_{3} \mathrm{mim}\right][\mathrm{Cl}]$} \\
\hline $\mathrm{NA}$ & 0.0343 & 0.0206 & 0.0348 \\
$\mathrm{CR}$ & -0.0031 & 0.0151 & -0.0553 \\
$\mathrm{NA}(\mathrm{m})$ & 0.0343 & -0.0134 & 0.0854 \\
$\mathrm{CW}(\mathrm{m})$ & -0.1620 & -0.0936 & -0.1756 \\
$\mathrm{CW}$ & -0.1620 & -0.2054 & -0.1508 \\
$\mathrm{H} 5$ & 0.1375 & 0.1249 & 0.1563 \\
$\mathrm{H} 4(\mathrm{~m})$ & 0.1909 & 0.1821 & 0.2037 \\
$\mathrm{H} 4$ & 0.1909 & 0.1889 & 0.1760 \\
$\mathrm{CT}$ & 0.2219 & 0.2394 & 0.2072 \\
$\mathrm{CT}$ & 0.2219 & 0.2075 & 0.1949 \\
$\mathrm{CT}$ & & 0.0373 & 0.0717 \\
$\mathrm{CT}$ & & & -0.0263 \\
\hline $\mathrm{Cl}$ & -0.7046 & -0.7035 & -0.7221 \\
\hline
\end{tabular}




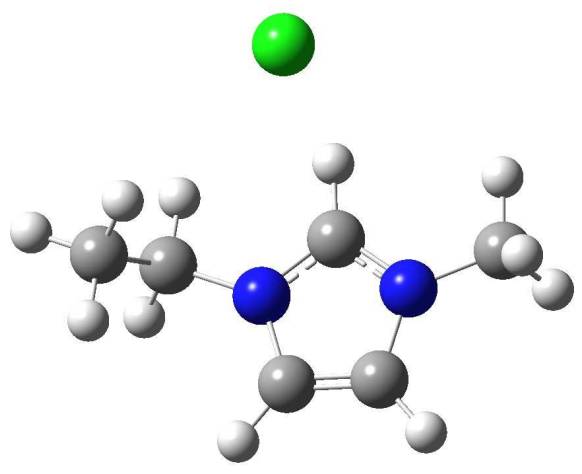

5e $(-0.723)$

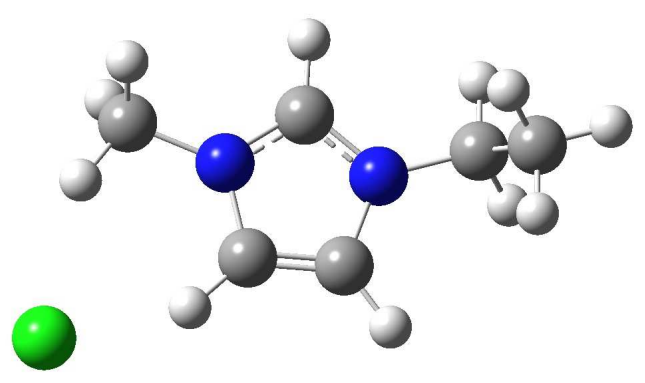

$4 \mathbf{m}(-0.824)$

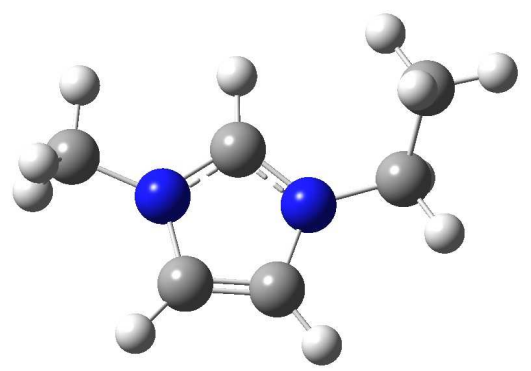

$5 \mathbf{m}(-0.751)$

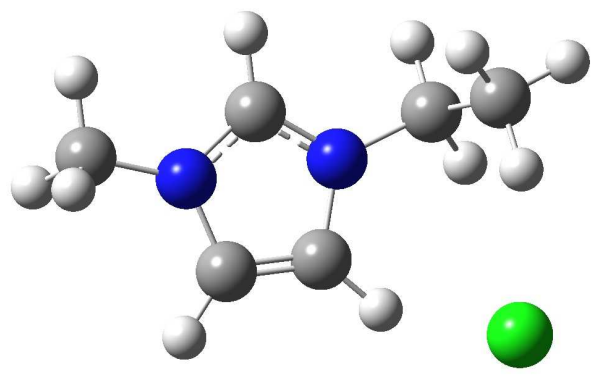

4 e $(-0.662)$

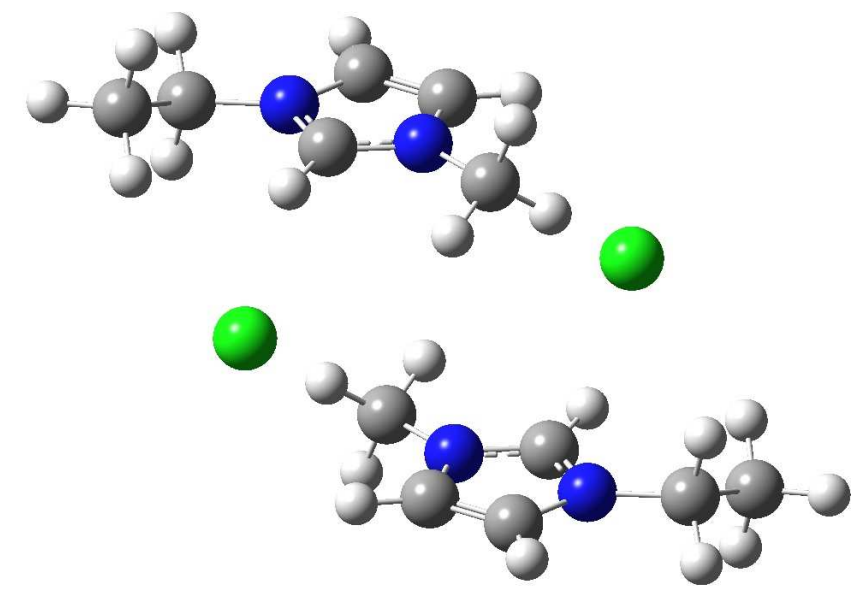

dimer

Figure S1. Four optimized isomers for $\left[\mathrm{C}_{2} \mathrm{mim}\right][\mathrm{Cl}]$ ion pair and its dimers, obtained by ab initio calculation at B3LYP/6-31+G(d,p) level. The charges on chloride are shown as the numbers in bracket. 


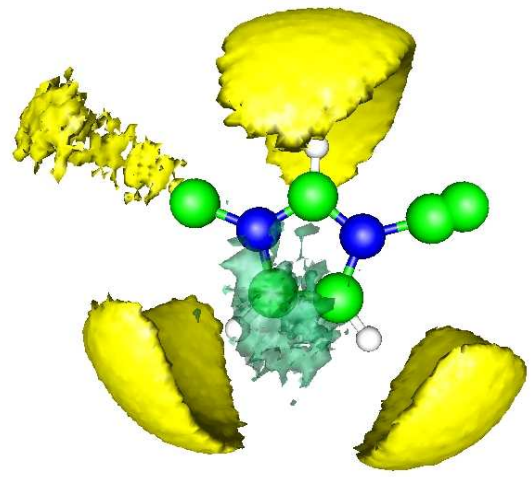

a.

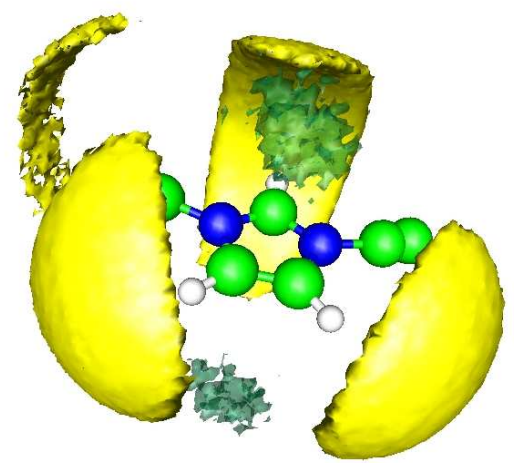

b

Figure S2. Three-dimensional probability distributions of chloride (yellow) and CR on cations (green) around cations in ionic liquid $\left[\mathrm{C}_{2} \mathrm{mim}\right][\mathrm{Cl}]$. Density levels correspond to five and three times the bulk density for anions and cations, respectively. Two different views are shown in a and b. 


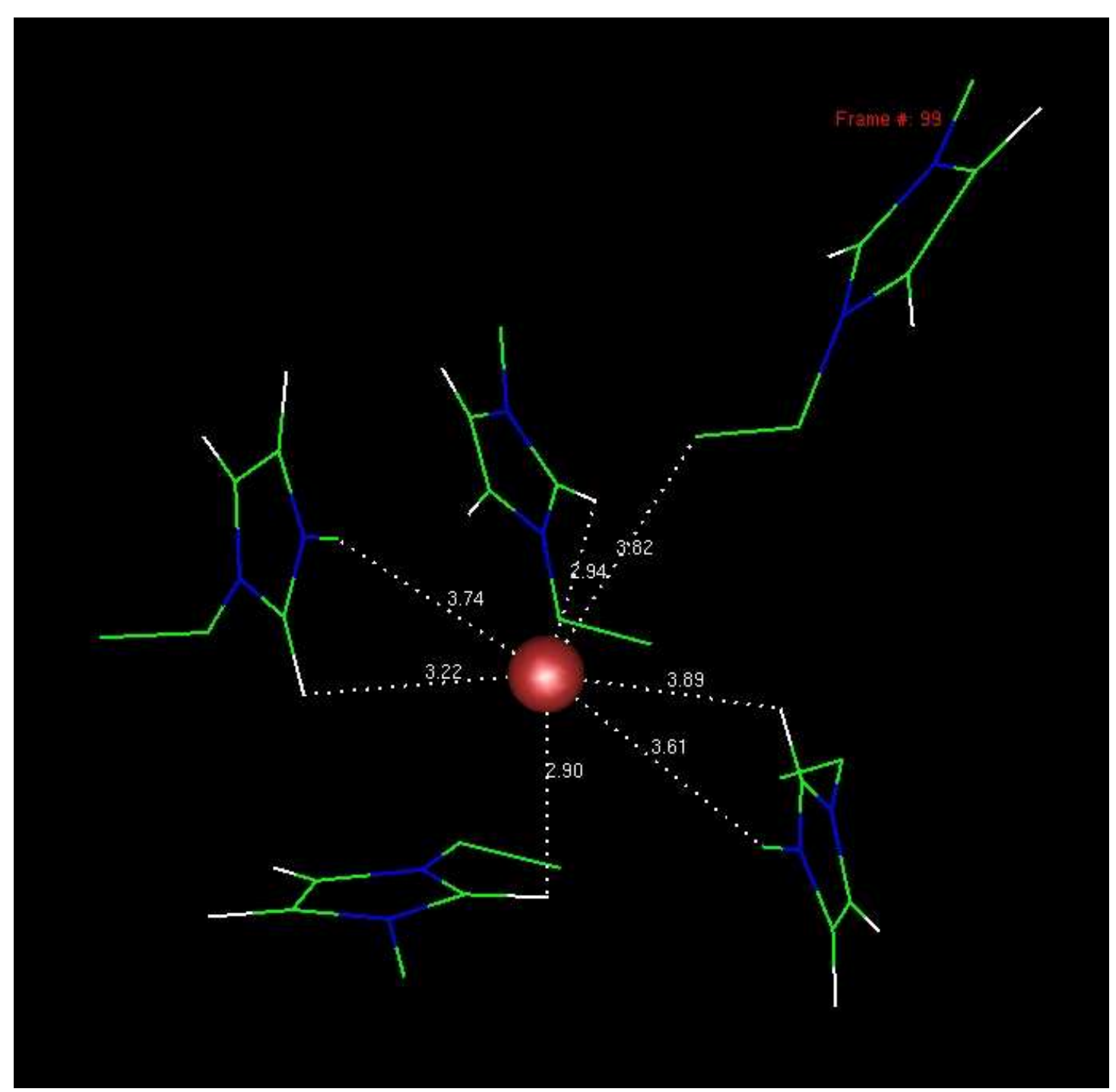

Figure S3. Typical coordination of cations around chloride in $\left[\mathrm{C}_{2} \mathrm{mim}\right][\mathrm{Cl}]$. Some short distances between chloride and hydrogen/carbon in cations are shown. 


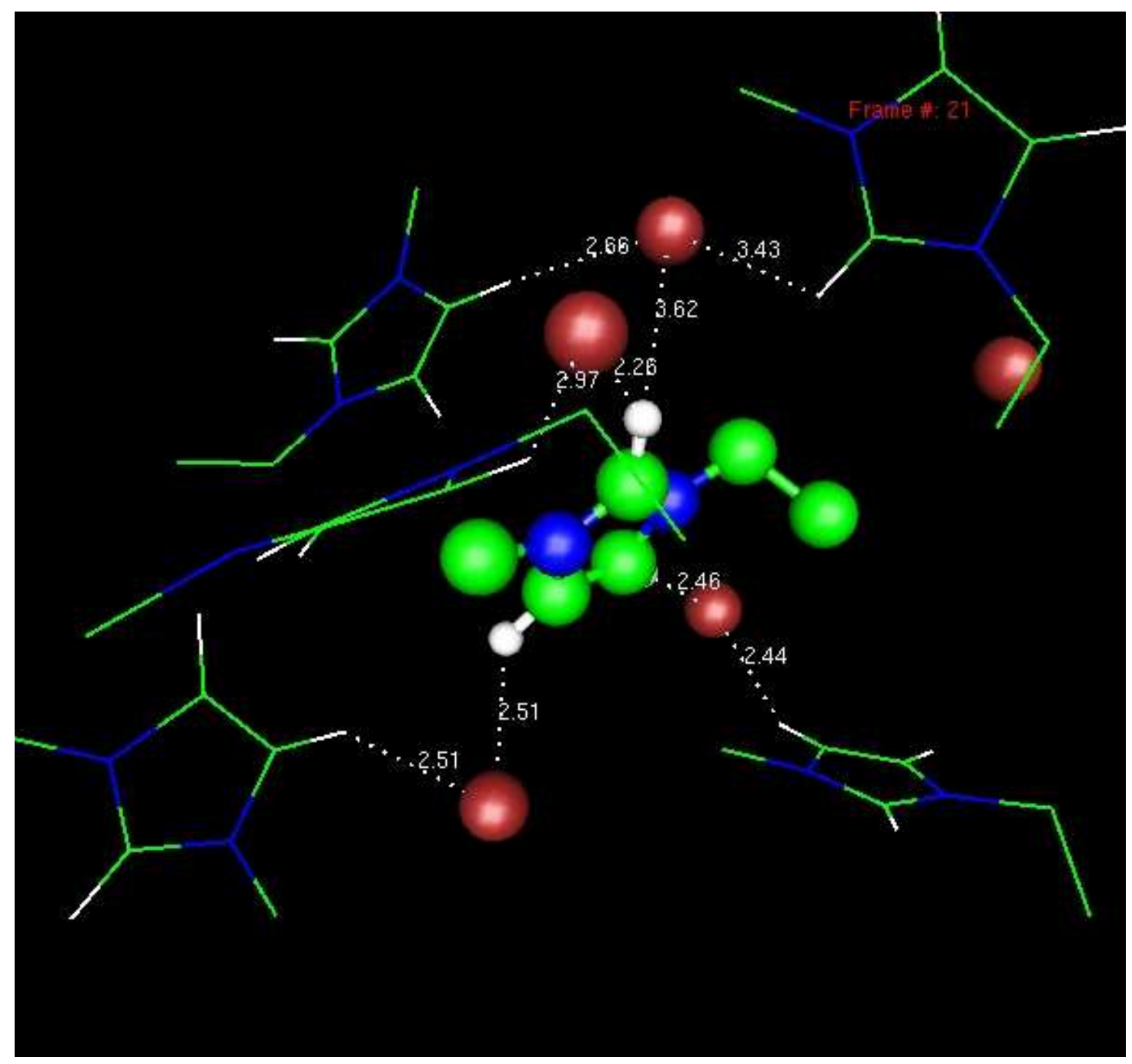

Figure S4. Typical coordination of choloride and cations around $\left[\mathrm{C}_{2} \mathrm{mim}\right]^{+}$in $\left[\mathrm{C}_{2} \mathrm{mim}\right][\mathrm{Cl}]$. Some short distances between chloride and hydrogen/carbon in cations are shown. 


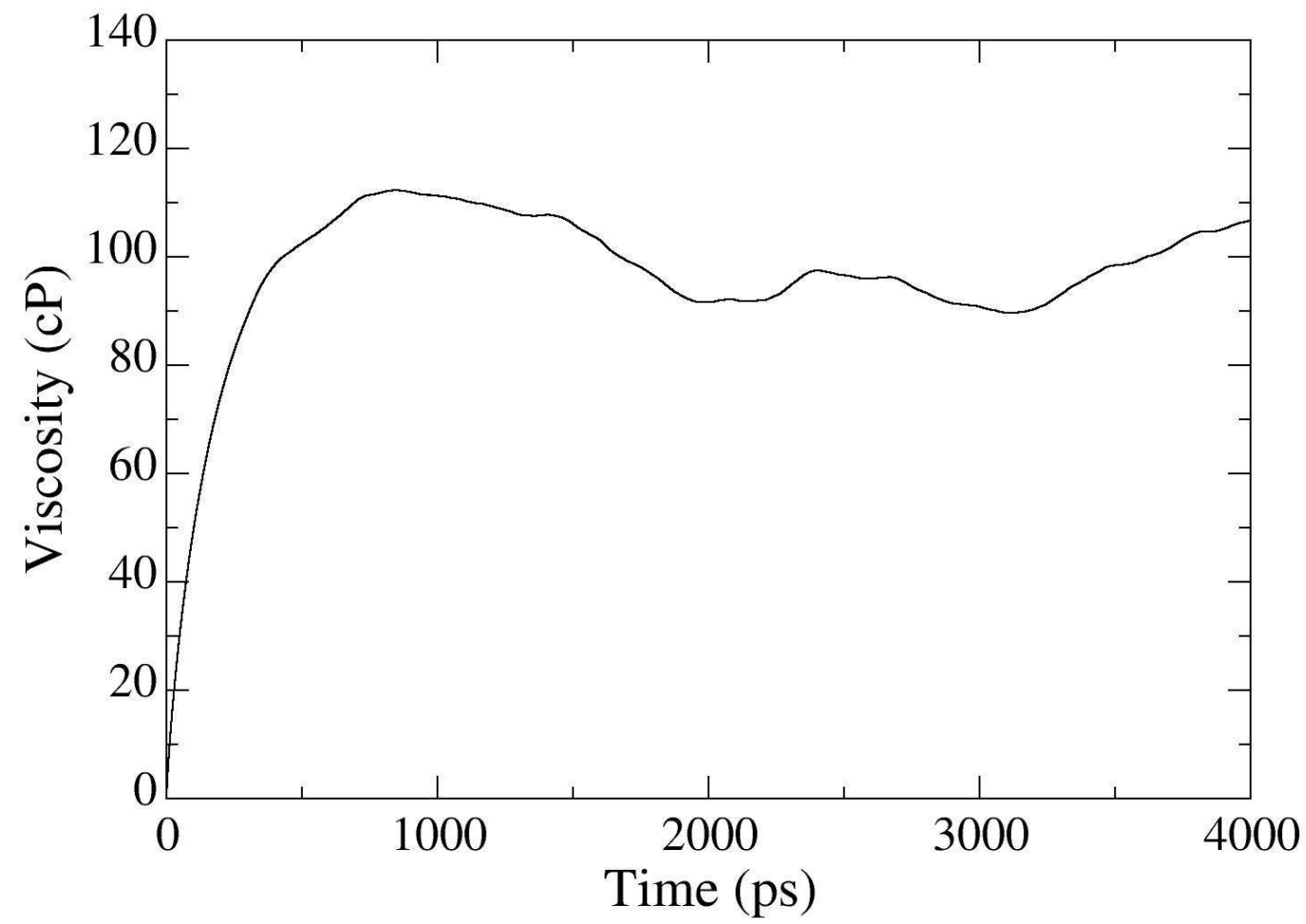

Figure S5 Viscosity of $\left[\mathrm{C}_{2} \mathrm{mim}\right][\mathrm{Cl}]$ at $353 \mathrm{~K}$ calculated by equation 6 in section 3.2.4. 